\title{
The role of nutritional indexes in predicting coronary artery disease severity in acute coronary syndrome
}

\author{
(1) Hatice Tolunay, (1) Suat Görmel, (1) Serkan Asil, (1) Salim Yaşar, (1) Erkan Yıldırım, (1) Ayşe Saatçi Yaşar, \\ (1) Murat Çelik, (1) Uygar Çağdaş Yüksel, (1) Cem Barçın
}

University of Health Sciences Turkey, Gülhane Training and Research Hospital, Clinic of Cardiology, Ankara, Turkey

\section{Date submitted: \\ 11.03.2021}

Date accepted:

13.04.2021

Online publication date:

15.06.2021

\section{Corresponding Author:}

Hatice Tolunay, M.D., University of Health Sciences Turkey, Gülhane Training and Research Hospital, Clinic of Cardiology, Ankara, Turkey

ORCID:

orcid.org/0000-0002-9407-3395

Keywords: Acute coronary syndrome, malnutrition, SYNTAX score

\begin{abstract}
Aims: The purpose of this study is to evaluate the association between nutritional status and coronary artery disease (CAD) severity in patients with acute coronary syndrome (ACS).

Methods: Patients with ACS who underwent coronary angiography were evaluated retrospectively. SYNTAX scores were calculated for each patient to determine the severity of CAD. The patients were divided into three groups based on their SYNTAX scores as low SYNTAX score ( $\leq 22)$, intermediate SYNTAX score (23-32) and high SYNTAX score ( $\geq 33)$. The Prognostic Nutritional Index (PNI) score was calculated with the formula using serum albumin and total lymphocyte count. The "The Control Nutritional Status (CONUT)" score was calculated by using three parameters which are serum albumin, total cholesterol and total lymphocyte count. Data on cardiovascular mortality and rehospitalization within six months of diagnosis were obtained from records.
\end{abstract}

Results: The study included 177 patients with ACS. The mean age of patients was $63.34 \pm 10.94$ years and $64.4 \%$ were male. Patients with high SYNTAX score had statistically significantly lower PNI score and higher CONUT score than patients with low and intermediate SYNTAX scores. There was a positive correlation between SYNTAX score and CONUT score $(r=0.256, p=0.002)$, and a negative correlation between SYNTAX score and PNI score $(r=-0.328, p<0.001)$. Within six months of ACS diagnosis, patients with cardiovascular mortality and rehospitalization had lower PNI scores and higher CONUT scores. According to multivariate logistic regression analysis, CONUT score was an independent predictor for high SYNTAX score (odds ratio=1.584; $95 \%$ confidence interval $=1.014-2.473 ; p=0.043$ ).

Conclusions: In our study, nutritional status assessed by PNI and CONUT scores was associated with the extent of coronary atherosclerosis in patients with ACS.

\section{Introduction}

Cardiovascular risk factors are becoming more common and the incidence of acute coronary syndrome (ACS) is increasing (1). Despite the advancement of percutaneous and surgical procedures, the mortality rate associated with ACS is still high $(2,3)$. Determining high-risk patients and especially modifiable risk factors is important in terms of primary prevention.

Malnutrition affects many people around the world and it is increasingly recognized as a potentially modifiable risk factor for cardiovascular diseases. Malnutrition is associated with poor prognosis in a wide variety of diseases including cancer $(4,5)$, renal disease requiring dialysis (6), end-stage liver disease (7) and heart failure (8-10). Malnutrition also influences adversely the prognosis of patients with ACS (11).

Prognostic Nutritional Index (PNI) is a score that reflects a person's nutritional status based on serum albumin and total lymphocyte count (4). It is an easy and effective method to calculate. A low score is associated with malnutrition. PNI has been associated with inflammatory processes in several studies. Recently, the prognostic value of $\mathrm{PNI}$ in various malignancies 
has been demonstrated (4). PNI is independently associated with long-term survival in patients hospitalized for heart failure with reduced or preserved ejection fraction $(8,10)$.

The Control Nutritional Status (CONUT) score is a score calculated by serum albumin level, total cholesterol level and total lymphocyte count (12). Albumin represents protein levels, total cholesterol represents lipid metabolism, and lymphocyte count represents immune function. A high score means that the nutritional status is poor (7). The studies on the CONUT score have described the relationship between mortality and prognosis after surgery in cancer patients (13).

The SYNTAX score is a useful angiographic scoring system for determining the severity of coronary artery disease (CAD). It represents the degree of atherosclerotic burden and complexity of coronary lesions (14). The relationship between cardiovascular risk factors such as diabetes mellitus and hypertension and the severity of atherosclerosis is known, but it remains unclear whether there is a relationship between nutritional status and CAD severity in ACS. Accordingly, in this study, we aimed to investigate the association between SYNTAX score and nutritional status assessed by PNI and CONUT scores, and to evaluate whether nutritional status influenced cardiovascular mortality and re-hospitalization within six months of diagnosis in patients with ACS.

\section{Methods}

Patients who underwent coronary angiography with the diagnosis of ACS at Gülhane Training and Research Hospital were evaluated retrospectively. Patients who had previously undergone percutaneous or surgical revascularization, patients with acute or chronic inflammatory disease, severe valvular heart disease, renal or hepatic insufficiency, malignancy, and patients with other major diseases that might affect serum albumin and total lymphocyte counts were excluded from the study. We also excluded patients taking statins or triglyceride (TG) lowering medication before the onset of ACS.

\section{Laboratory Measurements}

The recorded data of the patients were evaluated retrospectively and blood glucose, creatinine, total cholesterol, low-density lipoprotein (LDL) cholesterol, high-density lipoprotein cholesterol, TG, hemoglobin, white blood cell counts, and total lymphocyte counts were retrieved from the electronic database. All nutritional factors were measured on the day of hospitalization with the diagnosis of ACS.

\section{Evaluation of Nutritional Indexes}

The PNI scores showing the nutritional status of all patients were calculated and recorded with the formula: $10 \times$ serum albumin $(\mathrm{g} / \mathrm{dL})+0.005 \times$ total lymphocyte count $\left(\mathrm{mm}^{3}\right)(4)$.
CONUT score was calculated as shown in Table 1 based on previous studies $(7,12)$. The cut-off values were $3.5 \mathrm{~g} / \mathrm{dL}$ for serum albumin, $180 \mathrm{mg} / \mathrm{dL}$ for total cholesterol and $1600 / \mathrm{mm}^{3}$ for total peripheral lymphocyte count.

\section{Coronary Angiography}

Two interventional cardiologists without the knowledge of the data of the participants analyzed coronary angiograms and the SYNTAX scores of all patients were calculated with a series of questions through a computer program. The SYNTAX scoring system contains 12 basic questions. The first three questions cover the dominance, the total number of lesions, and the vessel segment where the lesions are located. The last nine questions are to identify adverse lesion characteristics. Scores for each lesion are calculated separately and the total SYNTAX score is obtained by summing all these scores. We divided patients into three groups based on their SYNTAX scores as low SYNTAX score $(\leq 22)$, intermediate SYNTAX score (23-32) and high SYNTAX score $(\geq 33)$ (14). Data on cardiovascular mortality and rehospitalization within six months of their diagnosis were obtained from hospital records and the database of the Ministry of Health.

\section{Statistical Analysis}

Data analysis was performed with Statistical Package for the Social Sciences (SPSS) for Windows, version 22.0 (SPSS Inc., Chicago, IL, USA). The distribution of continuous variables was determined using the Kolmogorov-Smirnov test. Continuous data were defined as mean \pm standard deviation for normal distributions and median (minimum-maximum value) for skewed distributions. Categorical data were defined as the number of cases (\%). Variables showing normal distribution between two independent groups were compared using the Student's t-test. Variables showing normal distribution between more than two independent groups were analyzed with the oneway ANOVA, and data not normally distributed were analyzed with the Kruskal-Wallis test. When the obtained $p$ value was statistically significant, LSD or Conover's non-parametric multiple comparison test was used to know which group was

\begin{tabular}{|c|c|c|c|c|}
\hline Parameters & Normal & Light & Moderate & Severe \\
\hline $\begin{array}{l}\text { Serum albumin } \\
(\mathrm{g} / \mathrm{dL})\end{array}$ & $\geq 3.5$ & $3.0-3.49$ & $2.5-2.99$ & $<2.5$ \\
\hline Score & 0 & 2 & 4 & 6 \\
\hline $\begin{array}{l}\text { Total cholesterol } \\
(\mathrm{mg} / \mathrm{dL})\end{array}$ & $\geq 180$ & $140-179$ & $100-139$ & $<100$ \\
\hline Score & 0 & 1 & 2 & 3 \\
\hline $\begin{array}{l}\text { Lymphocytes } \\
\left(/ \mathrm{mL}^{3}\right)\end{array}$ & $\geq 1600$ & $1200-1599$ & 800-1199 & $<800$ \\
\hline Score & 0 & 1 & 2 & 3 \\
\hline
\end{tabular}


different from others. Correlation degrees between variables were evaluated using the Pearson's correlation or Spearman correlation analysis.

Univariate ordinal logistic regression was used for risk factors thought to be associated with the SYNTAX score category. Risk factors with a one-variable logistic regression $p$ value of $<0.25$ were included in the multivariate logistic regression model. The significance of each independent variable on the model was analyzed using the Wald statistics. With Nagelkerke $R^{2}$, how much independent variable explained dependent variable was evaluated. A $p$ value of $<0.05$ was considered significant in all statistical analyses.

\section{Results}

Of the 550 patients who underwent coronary angiography with the diagnosis of ACS, 177 patients who met the inclusion criteria were included in the study. The mean age of the patients was $63.34 \pm 10.94$ years and $64.4 \%$ were male. The demographic characteristics, body mass index, laboratory findings, ejection fraction, PNI scores, CONUT scores and SYNTAX scores of the patients are shown in Table 2.

\begin{tabular}{|c|c|c|}
\hline \multicolumn{2}{|c|}{ Variable } & Overall \\
\hline \multicolumn{2}{|l|}{ Age, years } & $63.34 \pm 10.94$ \\
\hline \multicolumn{2}{|l|}{ Gender, male } & $114(64.4)$ \\
\hline \multicolumn{2}{|l|}{ BMI, $\mathrm{kg} / \mathrm{m}^{2}$} & $27.17 \pm 2.54$ \\
\hline \multicolumn{2}{|l|}{ Smoking } & $114(64.4)$ \\
\hline \multicolumn{2}{|l|}{ Hypertension } & $122(68.9)$ \\
\hline \multicolumn{2}{|c|}{ Diabetes mellitus } & $95(53.7)$ \\
\hline \multicolumn{2}{|c|}{ Hemoglobin, g/dL } & $13.47 \pm 1.96$ \\
\hline \multicolumn{2}{|c|}{ WBC count, $\times 10^{9} / L$} & $9.41 \pm 8.24$ \\
\hline \multicolumn{2}{|c|}{ Platelet count, $x 10^{9} / \mathrm{L}$} & $228.33 \pm 61.43$ \\
\hline \multicolumn{2}{|c|}{ Lymphocyte, x10\%/L } & $1.94 \pm 0.65$ \\
\hline \multicolumn{2}{|c|}{ Serum creatinine, $\mathrm{mg} / \mathrm{dL}$} & $1.10 \pm 0.66$ \\
\hline \multicolumn{2}{|l|}{ Albumin, g/dL } & $3.85 \pm 0.43$ \\
\hline \multicolumn{2}{|c|}{ Cholesterol, mg/dL } & $198.51 \pm 49.58$ \\
\hline \multicolumn{2}{|c|}{ HDL cholesterol, mg/dL } & $44.37 \pm 9.87$ \\
\hline \multicolumn{2}{|c|}{ LDL cholesterol, mg/dL } & $121.06 \pm 38.78$ \\
\hline \multicolumn{2}{|c|}{ Triglyceride, mg/dY } & $166.31 \pm 86.59$ \\
\hline \multicolumn{2}{|c|}{ LVEF (\%) } & $49.17 \pm 11.88$ \\
\hline \multicolumn{2}{|l|}{ PNI score } & $49.13 \pm 5.66$ \\
\hline \multicolumn{2}{|l|}{ CONUT score } & $1(0-7)$ \\
\hline \multicolumn{2}{|l|}{ SYNTAX score } & $19.71 \pm 10.32$ \\
\hline \multirow{3}{*}{ SYNTAX score } & Low $(\leq 22)$ & $61(34.5)$ \\
\hline & Intermediate (23-32) & $62(35.0)$ \\
\hline & High $(\geq 33)$ & $54(30.5)$ \\
\hline \multicolumn{3}{|c|}{$\begin{array}{l}\text { BMI: Body mass index, WBC: White blood cell, HDL: High-density lipoprotein, } \\
\text { LDL: Low-density lipoprotein, LVEF: Left ventricular ejection fraction, PNI: } \\
\text { Prognostic nutritional index, CONUT: Controlling Nutritional Status. } \\
\text { Continuous variables are expressed as either mean } \pm \text { standard deviation or } \\
\text { median (minimum-maximum). Categorical data are expressed as percentage. }\end{array}$} \\
\hline
\end{tabular}

The mean PNI scores of low, intermediate and high SYNTAX score groups were $51.05 \pm 4.99,49.89 \pm 5.17$, and $46.10 \pm 5.75$, respectively. A statistically significant difference was found between low and high SYNTAX score groups, and intermediate and high SYNTAX score groups in terms of PNI score ( $p<0.001$, for both) (Table 3$)$. The median CONUT scores of low, intermediate and high SYNTAX score groups were 1 (0$6), 1(0-7)$, and 2 (0-7), respectively. In terms of CONUT score, a statistically significant difference was found between low and high SYNTAX score groups, and intermediate and high SYNTAX score groups ( $p=0.001$ for both) (Table 3 ).

There was a low level of positive and statistically significant $(r=0.256, p=0.002)$ correlation between SYNTAX score and CONUT score, while there was a low level of negative and statistically significant $(r=-0.328, p<0.001)$ correlation between SYNTAX score and PNI score.

The mean serum albumin levels of low, intermediate and high SYNTAX score groups were $4.06 \pm 0.35,4.02 \pm 0.41$, and $3.71 \pm 0.43$, respectively. The difference was statistically significant between low and intermediate SYNTAX score groups, and intermediate and high SYNTAX score groups $(p<0.001$, for both). However, there was no significant difference in terms of total lymphocyte count in patients with low, intermediate and high SYNTAX score groups $(2.07 \pm 0.70,1.92 \pm 0.57,1.83 \pm 0.64$, $p=0.146)$.

Within six months of ACS diagnosis, patients with cardiovascular mortality and re-hospitalization had lower PNI scores and higher CONUT scores. A statistically significant difference was found in PNI score and CONUT score in all paired group comparisons ( $p<0.001$, for all) (Table 4).

Patients with low SYNTAX score were included as the reference category in the logistic regression analysis. Diabetes mellitus was independent predictor for intermediate [odds ratio $(\mathrm{OR})=2.288$; 95\% confidence interval $(\mathrm{Cl})=1.110-4.713$; $\mathrm{p}=0.025]$ and high SYNTAX scores $(\mathrm{OR}=4.329 ; 95 \% \mathrm{Cl}=1.734-$ 10.803; $p=0.002)$. LDL cholesterol (OR=1.015; 95\% Cl=1.0021.028; $p=0.021)$ and CONUT score $(\mathrm{OR}=1.584 ; 95 \% \mathrm{Cl}=1.014-$ 2.473; $p=0.043$ ) were independent predictors for high SYNTAX score (Table 5).

\begin{tabular}{|c|c|c|c|c|}
\hline & \multicolumn{3}{|c|}{ SYNTAX score } & \multirow[b]{2}{*}{$\mathbf{p}$} \\
\hline & $\begin{array}{l}\text { Low } \\
\leq 22\end{array}$ & $\begin{array}{c}\text { Intermediate } \\
23-32\end{array}$ & $\begin{array}{l}\text { High } \\
\geq 33\end{array}$ & \\
\hline PNI score* & $51.05 \pm 4.99$ & $49.89 \pm 5.17$ & $46.10 \pm 5.75$ & $<0.001^{a, b}$ \\
\hline $\begin{array}{l}\text { CONUT } \\
\text { score }^{\beta}\end{array}$ & $1(0-6)$ & $1(0-7)$ & $2(0-7)$ & $0.001^{\mathrm{a}, \mathrm{b}}$ \\
\hline \multicolumn{5}{|c|}{$\begin{array}{l}\text { Continuous variables are expressed as either }{ }^{*} \text { mean } \pm \text { standard deviation or } \\
\beta \text { median (minimum-maximum). Continuous variables were compared with } \\
{ }^{*} \text { One-Way ANOVA test or }{ }^{\beta} \text { Kruskal-Wallis test and the } p \text { value was set at } 0.05 \text {. } \\
\text { Significant differences were found between; }{ }^{\text {a: }} \text { Low vs. High, b: Intermediate vs. } \\
\text { High. } \\
\text { CONUT: Controlling Nutritional Status, PNI: Prognostic Nutritional Index }\end{array}$} \\
\hline
\end{tabular}




\section{Discussion}

In our study, PNI and CONUT scores were significantly associated with SYNTAX score and CONUT score was an independent predictor for high SYNTAX score in patients with ACS. Besides, PNI and CONUT scores were associated with cardiovascular mortality and rehospitalization within six months of ACS diagnosis.

There are different nutritional scores to define malnutrition. The PNI and CONUT scores both involve the measurement of serum albumin level and total lymphocyte count. Unlike the PNI score, the CONUT score also takes into account serum cholesterol. The main protein in the blood is albumin and it is the determinant of oncotic pressure (15). Albumin is an acute phase reactant that can be reduced by decreased synthesis or increased loss in inflammation, liver diseases, renal failure and some gastrointestinal tract diseases. Low serum albumin level has been reported to be associated with poor prognosis in patients with heart failure (16). In our study, serum albumin levels were lower in patients with high SYNTAX scores than low and intermediate SYNTAX scores. Kurtul et al. (17) examined the association between serum albumin level and severity of CAD as measured by SYNTAX score in patients with ACS. They found that serum albumin level was inversely associated with high SYNTAX score and in-hospital mortality in ACS. High serum albumin level can be considered as a protective shield in acute events.

\begin{tabular}{|c|c|c|c|c|}
\hline & Stable & Re-hospitalization & Death & $\mathbf{p}$ \\
\hline PNI score* & $50.57 \pm 4.63$ & $44.84 \pm 4.05$ & $38.90 \pm 5.27$ & $<0.001^{a, b, c}$ \\
\hline CONUT score ${ }^{\beta}$ & $1(0-6)$ & $2(0-6)$ & $5(2-7)$ & $<0.001^{a, b, c}$ \\
\hline \multicolumn{5}{|c|}{ 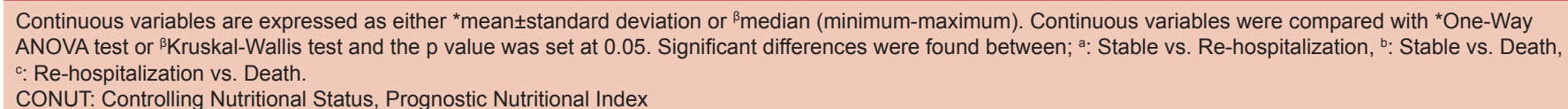 } \\
\hline
\end{tabular}

Table 5. Predictors of intermediate and high SYNTAX scores with logistic regression analysis

\begin{tabular}{|c|c|c|c|c|c|c|c|c|}
\hline & \multicolumn{4}{|c|}{ Univariate analysis } & \multicolumn{4}{|c|}{ Multivariate analysis } \\
\hline & Wald & OR & $95 \% \mathrm{Cl}$ & p & Wald & OR & $95 \% \mathrm{Cl}$ & p \\
\hline \multicolumn{9}{|c|}{ Intermediate SYNTAX score } \\
\hline BMI & 0.672 & 1.056 & $0.927-1.202$ & 0.412 & & & & \\
\hline HT & 0.004 & 1.024 & $0.482-2.178$ & 0.950 & & & & \\
\hline DM & 5.034 & 2.288 & $1.110-4.713$ & 0.025 & 5.034 & 2.288 & $1.110-4.713$ & 0.025 \\
\hline Smoking & 1.019 & 1.468 & $0.697-3.093$ & 0.313 & & & & \\
\hline Cholesterol & 0.001 & 1.000 & 0.992-1.008 & 0.971 & & & & \\
\hline LDL-C & 0.221 & 1.002 & 0.993-1.012 & 0.639 & & & & \\
\hline HDL-C & 0.609 & 0.985 & 0.949-1.023 & 0.435 & & & & \\
\hline TG & 0.127 & 1.001 & 0.997-1.005 & 0.722 & & & & \\
\hline PNI score & 1.578 & 0.955 & 0.890-1.026 & 0.209 & & & & \\
\hline CONUT score & 0.593 & 0.892 & $0.852-1.443$ & 0.441 & & & & \\
\hline \multicolumn{9}{|c|}{ High SYNTAX score } \\
\hline BMI & 0.334 & 1.044 & 0.9021 .210 & 0.563 & & & & \\
\hline HT & 0.339 & 1.268 & $0.570-2.823$ & 0.560 & & & & \\
\hline DM & 10.523 & 3.596 & $1.660-7.792$ & 0.001 & 9.860 & 4.329 & $1.734-10.803$ & 0.002 \\
\hline Smoking & 0.065 & 1.103 & $0.519-2.344$ & 0.799 & & & & \\
\hline Cholesterol & 0.796 & 1.003 & 0.996-1.011 & 0.372 & & & & \\
\hline LDL-C & 2.709 & 1.008 & 0.998-1.018 & 0.100 & 5.340 & 1.015 & $1.002-1.028$ & 0.021 \\
\hline HDL-C & 0.052 & 0.996 & $0.961-1.032$ & 0.819 & & & & \\
\hline TG & 0.529 & 0.998 & 0.994-1.003 & 0.467 & & & & \\
\hline PNI score & 17.093 & 0.839 & $0.772-0.912$ & $<0.001$ & 3.116 & 0.902 & 0.804-1.011 & 0.078 \\
\hline CONUT score & 12.598 & 1.685 & $1.263-2.248$ & $<0.001$ & 4.092 & 1.584 & $1.014-2.473$ & 0.043 \\
\hline
\end{tabular}

Cl: Confidence interval, BMI: Body mass index, HT: Hypertension, DM: Diabetes mellitus, LDL-C: Low-density lipoprotein-cholesterol, HDL-C: High-density lipoproteincholesterol, OR: Odds ratio, TG: Triglyceride, CONUT: Controlling Nutritional Status, PNI: Prognostic Nutritional Index. Reference category: Low; *OR is statistically significant ( $\mathrm{Cl}$ does not include 1); Variables with $p$ value $<0.25$ in univariant analysis were evaluated by multivariant analysis; $p$ value $<0.05$ as significant level 
The lymphocyte count decreases as a stress response in patients with ACS (18) and lymphopenia has been reported to be an early marker of myocardial infarction (19). While the lymphocyte count decreases, the peripheral neutrophil count increases in acute myocardial infarction and neutrophil/ lymphocyte ratio predicts long term mortality in these patients (20). Further, Arbel et al. (21) demonstrated the correlation between neutrophil/lymphocyte ratio and severity of CAD in a cohort undergoing coronary angiography. In our study, no difference was found in total lymphocyte count between SYNTAX groups. In the review of the literature, we did not find any study clarifying whether there was a direct relationship between total lymphocyte count and CAD severity.

It has been demonstrated that malnutrition contributes to poor prognosis after acute coronary events. Yoo et al. (22) showed an association between undernutrition and poor clinical outcomes during hospitalization in patients with acute myocardial infarction. Recently, Raposeiras Roubín et al. (11) investigated the prevalence and prognostic effects of malnutrition in patients with ACS by using three different indexes; PNI score, Nutritional Risk Index and CONUT score. They found that malnutrition was prevalent in patients with ACS and was associated with all-cause mortality and major cardiovascular events including cardiovascular death, reinfarction or ischemic stroke. Their explanation for the poor prognosis of malnutrition in patients with ACS is that nutritional condition may be a proxy indicator of inflammation and a trigger for progression of atherosclerosis and plaque rupture. As inflammation and malnutrition occur concomitantly and these two conditions are closely associated with atherosclerosis, they have been recently referred together as "malnutrition-inflammation-atherosclerosis syndrome" (23).

The predictive value of PNI score for long term cardiovascular outcomes has also been reported in stable CAD patients (24). Chen et al. (25) investigated the predictive value of CONUT score in CAD patients undergoing percutaneous coronary intervention including stable and ACS patients. They found that an increased CONUT score was associated with adverse events after percutaneous coronary intervention. In the present study, CONUT score was an independent predictor for high SYNTAX score in patients with ACS. The combination of three parameters in the CONUT score may more accurately reflect immune and nutritional conditions. However, there is no consensus on which nutritional index should be used for patients with ACS. Regardless of which index is used, early recognition and treatment of malnutrition may provide improvement in many cardiovascular diseases. These nutritional indexes can help physicians to predict the extent and complexity of CAD.

Our study has some limitations. The study has a crosssectional and retrospective design, so we do not have data showing the long term prognostic value of $\mathrm{PNI}$ and CONUT score rather than six-month data. With prospective studies including larger patient populations, the relationship between CAD and PNI, CONUT score, and long term prognostic clinical significance can be clarified.

\section{Conclusion}

In the present study, PNI and CONUT scores associate with the severity of CAD assessed by SYNTAX score in ACS. Adequate assessment of the nutritional status is important for identifying patients at high risk for CAD and selecting patients who can benefit from nutritional support and risk modification strategies. Our data should be supported by large-scale multicenter studies, including patients with stable angina pectoris.

\section{Ethics}

Ethics Committee Approval: The study was approved by the Ankara City Hospital Ethics Committee (date: 12/16/2020, decision no: E2-20-90).

Informed Consent: Retrospective study.

Peer-review: Externally peer-reviewed.

\section{Authorship Contributions}

Concept: H.T., S.G., A.S.Y., Design: H.T., M.Ç., C.B., Data Collection or Processing: H.T., S.G., S.A., S.Y., Analysis or Interpretation: H.T., M.Ç., C.B., Literature Search: H.T., S.G., S.A., S.Y., U.Ç.Y., Writing: H.T., E.Y., M.Ç., A.S.Y.

Conflict of Interest: No conflict of interest was declared by the authors.

Financial Disclosure: The authors declared that this study received no financial support.

\section{References}

1. Szummer $\mathrm{K}$, Jernberg $\mathrm{T}$, Wallentin $\mathrm{L}$. From early pharmacology to recent pharmacology interventions in acute coronary syndromes: JACC state-of-the-art review. J Am Coll Cardiol. 2019;74:1618-1636.

2. Puymirat E, Simon T, Steg PG, et al. Association of changes in clinical characteristics and management with improvement in survival among patients with ST-elevation myocardial infarction. JAMA. 2012;308:998-1006.

3. Neumann FJ, Sousa-Uva M, Ahlsson A, et al. 2018 ESC/ EACTS Guidelines on myocardial revascularization. Eur Heart J. 2019;40:87-165.

4. Sun K, Chen S, Xu J, et al. The prognostic significance of the prognostic nutritional index in cancer: a systematic review and meta-analysis. J Cancer Res Clin Oncol. 2014;140:1537-1549.

5. Onodera T, Goseki N, Kosaki G. Prognostic nutritional index in gastrointestinal surgery of malnourished cancer patients. Nihon Geka Gakkai Zasshi. 1984;85:1001-1005.

6. Kobayashi I, Ishimura E, Kato Y, et al. Geriatric Nutritional Risk Index, a simplified nutritional screening index, is a 
significant predictor of mortality in chronic dialysis patients. Nephrol Dial Transplant. 2010;25:3361-3365.

7. Fukushima K, Ueno $\mathrm{Y}$, Kawagishi $\mathrm{N}$, et al. The nutritional index 'CONUT' is useful for predicting long-term prognosis of patients with end-stage liver diseases. Tohoku J Exp Med. 2011;224:215-219.

8. Cheng YL, Sung SH, Cheng HM, et al. Prognostic nutritional Index and the risk of mortality in patients with acute heart failure. J Am Heart Assoc. 2017;6:e004876.

9. Narumi T, Arimoto T, Funayama A, et al. Prognostic importance of objective nutritional indexes in patients with chronic heart failure. J Cardiol. 2013;62:307-313.

10. Zencirkiran Agus H, Kahraman S. Prognostic nutritional index predicts one-year outcome in heart failure with preserved ejection fraction. Acta Cardiol. 2020;75:450-455.

11. Raposeiras Roubín S, Abu Assi E, Cespón Fernandez M, et al. Prevalence and prognostic significance of malnutrition in patients with acute coronary syndrome. J Am Coll Cardiol. 2020;76:828-840.

12. Ignacio de Ulíbarri J, González-Madroño A, de Villar NG, et al. CONUT: a tool for controlling nutritional status. First validation in a hospital population. Nutr Hosp. 2005;20:3845.

13. Yoshida N, Harada K, Baba $\mathrm{Y}$, et al. Preoperative controlling nutritional status (CONUT) is useful to estimate the prognosis after esophagectomy for esophageal cancer. Langenbecks Arch Surg. 2017;402:333-341.

14. Sianos G, Morel MA, Kappetein AP, et al. The SYNTAX Score: an angiographic tool grading the complexity of coronary artery disease. Eurointervention. 2005;1:219227.

15. Doweiko JP, Nompleggi DJ. Role of albumin in human physiology and pathophysiology. JPEN J Parenter Enteral Nutr. 1991;15:207-211.

16. Horwich TB, Kalantar-Zadeh K, MacLellan RW, Fonarow GC. Albumin levels predict survival in patients with systolic heart failure. Am Heart J. 2008;155:883-889.
17. Kurtul A, Murat SN, Yarlioglues M, et al. Usefulness of Serum Albumin Concentration to Predict High Coronary SYNTAX Score and In-Hospital Mortality in Patients With Acute Coronary Syndrome. Angiology. 2016;67:34-40.

18. Ommen SR, Gibbons RJ, Hodge DO, Thomson SP. Usefulness of the lymphocyte concentration as a prognostic marker in coronary artery disease. Am J Cardiol. 1997;79:812-814.

19. Thomson SP, Gibbons RJ, Smars PA, et al. Incremental value of the leukocyte differential and the rapid creatine kinase-MB isoenzyme fort he early diagnosis of myocardial infarction Ann Int Med. 1995;122:335-341.

20. Núñez J, Núñez E, Bodí V, et al. Usefulness of the neutrophil to lymphocyte ratio in predicting long-term mortality in ST segment elevation myocardial infarction. Am J Cardiol. 2008;101:747-752.

21. Arbel Y, Finkelstein A, Halkin A, et al. Neutrophil/lymphocyte ratio is related to the severity of coronary artery disease and clinical outcome in patients un- dergoing angiography. Atherosclerosis. 2012;225:456-460.

22. Yoo SH, Kook HY, Hong YJ, Kim JH, Ahn Y, Jeong MH. Influence of undernutrition at admission on clinical outcomes in patients with acute myocardial infarction. J Cardiol. 2017;69:555-560.

23. Sueta D, Hokimoto S, Sakamoto K, etal. Validation of the high mortality rate of malnutrition-inflammation-atherosclerosis syndrome:-Community based observational study. Int J Cardiol. 2017;230:97-102.

24. Wada H, Dohi T, Miyauchi K, et al. Relationship between the prognostic nutritional index and long-term clinical outcomes in patients with stable coronary artery disease. J Cardiol. 2018;72:155-161.

25. Chen SC, Yang $\mathrm{YL}, \mathrm{Wu} \mathrm{CH}$, et al. Association between preoperative nutritional status and clinical outcomes of patients with coronary artery disease undergoing percutaneous coronary intervention. Nutrients. 2020;12:1295. 